\title{
Design, sensing and control of a robotic prosthetic eye for natural eye movement
}

\author{
J. J. Gu' ${ }^{1}$ M. Meng ${ }^{2}$, A. Cook ${ }^{3}$ and P. X. Liư \\ ${ }^{1}$ Department of Electrical and Computer Engineering, Dalhousie University, Halifax, NS, Canada B37 2 X4 \\ ${ }^{2}$ Department of Electronic Engineering, The Chinese University of Hong Kong, Shatin, Hong Kong, \\ People's Republic of China \\ ${ }^{3}$ Dean of Faculty of Rehabilitation Medicine, University of Alberta, 3-48 Corbett Hall, Edmonton, AB, \\ Canada T6G $2 G 4$ \\ ${ }^{4}$ Department of Systems and Computer Engineering, Carleton University, Ottama, ON, Canada K1S $5 B 6$
}

\begin{abstract}
Loss of an eye is a tragedy for a person, who may suffer psychologically and physically. This paper is concerned with the design, sensing and control of a robotic prosthetic eye that moves horizontally in synchronization with the movement of the natural eye. Two generations of robotic prosthetic eye models have been developed. The first generation model uses an external infrared sensor array mounted on the frame of a pair of eyeglasses to detect the natural eye movement and to feed the control system to drive the artificial eye to move with the natural eye. The second generation model removes the impractical usage of the eye glass frame and uses the human brain EOG (electro-oculargraph) signal picked up by electrodes placed on the sides of a person's temple to carry out the same eye movement detection and control tasks as mentioned above. Theoretical issues on sensor failure detection and recovery, and signal processing techniques used in sensor data fusion, are studied using statistical methods and artificial neural network based techniques. In addition, practical control system design and implementation using micro-controllers are studied and implemented to carry out the natural eye movement detection and artificial robotic eye control tasks. Simulation and experimental studies are performed, and the results are included to demonstrate the effectiveness of the research project reported in this paper.
\end{abstract}

Key words: Robotic eye, sensing and control, biomedical signal, sensor failure detection, electrode placement.

\section{INTRODUCTION}

Loss of an eye is a tragedy for a person, who may suffer psychologically and physically. The reconstructive surgeons can replace the lost eye with an ocular implant (Martin and Clodius 1979; Kandel et al. 1995). However, the implant is static. The purpose of our project is to develop an ocular system to let the artificial eye have the same natural movement. To provide the artificial eye with the same functionality as the muscles, the artificial eye is mounted onto a tiny small servomotor. The aim of the present project is to

Corresponding Author:

J. J. Gu

Department of Electrical and Computer Engineering

Dalhousie University

Halifax, NS

Canada B3J 2X4

Email: jason.gu@dal.ca sense the natural eye movement in the horizontal direction only and then to control the motor to drive the artificial eyeball to move correspondingly and naturally, matching the horizontal movement of the natural eye. Eye movement methods have been extensively reviewed to find a suitable approach for this project. For eye movement sensing, the following methods have been reviewed: electrodes, magnetic induction, optical sensing, photoelectric methods, infrared oculography (IROG) and video imaging. To find a suitable sensor, some constraints have to be considered, e.g., the sensor should be small, easy to mount, not invasive and not obstruct the vision of the real eye.

From these techniques, two suitable sensing techniques for the robotic eye system are identified.

The first one is an infrared reflection technique. The infrared emitter will send out the infrared light to illuminate the artificial eye, and an infrared detector will receive the reflected infrared light. The relationship between the infrared array output and the eye position is non-linear. Here 
we use a neural network based sensor fusion technique to detect the eye movement.

The second approach is electro-ocular-graph (EOG)based sensing. As a classic eye movement method, EOG is extensively used for many applications, such as detection of the rapid eye movement in infants (Barschdorff and Gerhardt 1996), determination of the eye position in fast jet flight (Viveash et al. 1994a) and in the development of a fish-eye VR system (Yagi et al. 1996). There are many techniques used to analyse EOG signal, such as the Kalman filter (Sauter et al. 1991), conventional and matched filtering methods (Hatzilabrou et al. 1994) and the non-linear eye movement detection method (Varri et al. 1996).

EOG signal is always corrupted by the eye blink signal, which is of high amplitude with a short half-wave lasting approximately $0.2 \mathrm{~s}$ (Varri et al. 1996). The addressed problem can be solved through using the median filter, which has been previously applied to biological signal processing, mainly to situations in which the mean value of the signal abruptly changes.

The rest of the paper is organized as follows. In the 'Methods', the neural network based sensor fusion method and the EOG-based eye movement detection method are described, also the median filter and improved median filter are given and their characteristics are explored to remove the eye blink corrupted signal from the EOG signal. In the section 'Technical aspects of experimental system', the technical aspects of the experimental system are given. The section 'Experimental results' presents the results of both methods. Conclusions are given at the end of paper.

\section{METHODS}

\section{Neural network-based sensor fusion and fault detection and recovery in robotic eye system}

This section describes an approach of using the artificial neural network to carry out sensor fusion to detect the eye movement in the robotic eye system.

Two types of neural networks are used for the sensor fusion and sensor fault detection and recovery, respectively. Usually the sensor fusion relies on the model of the system, however, sometimes it is not possible to get the accurate model of the system, or one or several of the parameters of the system may be unknown or partially known. In addition, there may be measurement inaccuracies associated with the sensors. In this case, the conventional method may not give a good performance. An artificial neural network can learn the characteristic of a non-linear, nonmodelled system through training samples. Then, during real application, the sensor signal can be used to feed the network and obtain the desired output, using the microsensor array to detect the eye movement carried out in the experimental study. The sensor signal is amplified, digitized and then sent to the computer. Two-layer neural networks are trained using data samples. The first trained network is used for sensor fusion, and the second two neural networks are used to detect the sensor failure and recover the faulty data. Experimental studies in terms of soft sensor failure and hard sensor failure are included.

Multisensor integration and fusion have received much attention in recent years (Hackett and Shah 1990). The Bayesian method is one of the classic methods that have been used in sensor fusion. This method has its short comings. It lacked flexibility and could not discriminate between uncertainty and ignorance. Thus Dempster-Shafer theory is used to overcome this shortcoming, as described in Jung et al. (1996) and Garvey et al. (1981). It provided a way for information fusion where uncertain elements existed. Instead of placing an exact probability on a given event as the Bayesian theory did, upper and lower probabilities are used as likelihood bounds. It is used for image processing and signal classification. Based on the Bayesian theory and Dempster-Shafer theory, Wang et al. (1996) presented a new strategy for statistical decision and evidence combination, which is called double-bound testing (DBT): this increased the flexibility of decision. All of the above methods are statistically based on fusion algorithms, which have been successfully applied to sensor fusion in some applications. However, statistically based fusion algorithm policies need a priori knowledge about the observation process to make inference about identity, which is sometimes difficult to obtain. To compensate for this drawback, neural network and fuzzy set based fusion policies are distribution free and no prior knowledge is needed about the statistical distributions of data in order to apply these methods for fusion.

The neural network is used to carry out motion detection (Yuan et al. 1996), object detection (Zheng and Bhanu 1996), speech perception (Kabre 1996) and signal processing (Chung and Merat 1996).

Lee (1996) presented a perception-action network. The net embedded feasible system behaviours in various levels of abstraction, so that the system can re-plan and control its behaviours towards the set goals. The fuzzy approach (Wide and Driankov 1996) is used for classification.

An artificial neural network can learn the characteristics of a non-linear, non-modelled system through training samples. Then during the real application, the sensor signal can be used to feed the network and obtain the desired output.

\section{Two-layer neural network}

An artificial neural network can learn the characteristics of a non-linear, non-modelled system through training samples. Assume there are $n$ inputs $X=\left[x_{1}, x_{2}, \ldots, x_{n}\right]$ and $m$ outputs $Y=\left[y_{1}, y_{2}, \ldots, y_{m}\right]$. They are related by a nonlinear unknown function $Y=F(X)$. A neural network, sketched in Fig. 1, is able to learn the relationship between $X$ and $Y$.

This is a two-layer neural network. The input layer is a hidden layer, and all the neurons are fed with the sensor measurement $x_{i}$, where $i$ varies from 1 to $n$. 


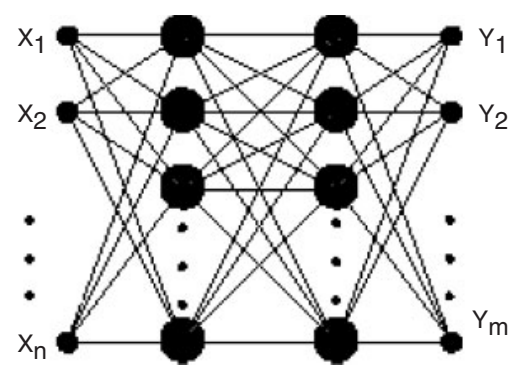

Figure 1 Two-layer neural network.

The hidden neurons have activation function $F_{\text {in }}$ and biases $B_{\text {in }}$.

The output layer is the second layer, and all the neurons of this layer are fed with the output of the hidden layer. The output neurons have activation function $F_{\text {out }}$ and biases $B_{\text {out }}$.

A set of weights is connected to each layer. Let $W_{\text {in }}$ be the hidden layer and $W_{\text {out }}$ the output layer.

In this network, the output of $Y$ can be expressed as

$$
Y=F_{\text {out }}\left(W_{\text {out }} F_{\text {in }}\left(W_{\text {in }} X\right)+B_{\text {in }}\right)+B_{\text {out }}
$$

\section{Neural network for learning}

A supervised learning method is used for this two-layer network. The output of the network is compared to the desired output. The error is used to adjust the weight and the bias. In this way, the network can be trained by minimizing this error term. The block diagram of the learning method is shown in Fig. 2.

\section{Sensor fusion and sensor failure detection}

Figure 3 is the block diagram for sensor fusion and detection. $D_{i j}$ is $j$ th data of sensor $i$. Each row of the data is the successive data from same sensor, and each column of the data is the data from the entire sensor at the same time. Each row of data and the time interval are fed into the neural network to obtain the weight, and the estimated sensor output can be acquired. The last column is the newest data, which is used as the input for the trained neural network.

\section{EOG-based robotic eye system}

This section describes another approach using EOG to detect the eye movement signal. The basis for this system is

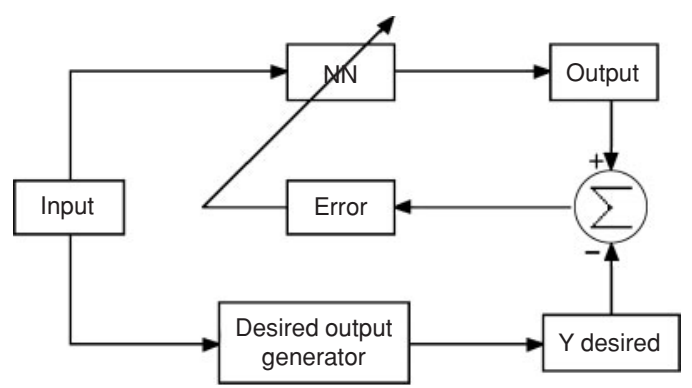

Figure 2 Supervised learning.

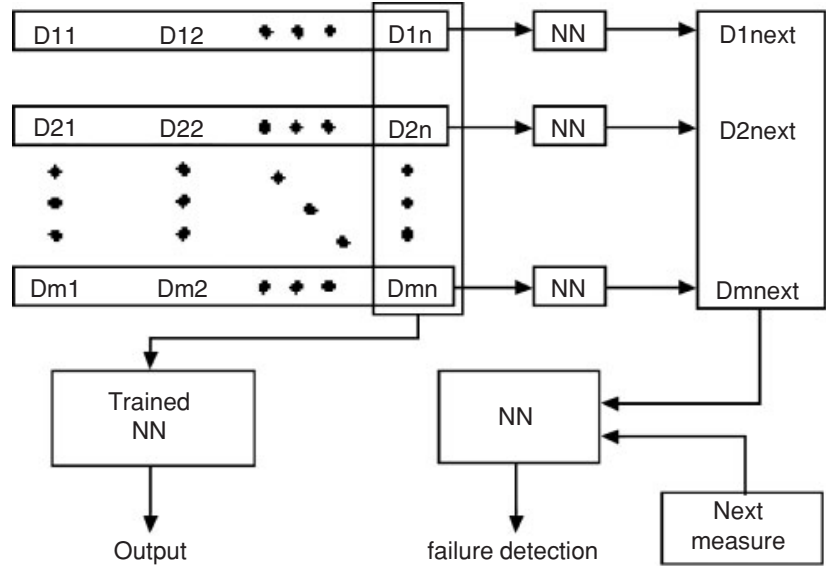

Figure 3 Fusion and sensor failure detection.

the use of bioelectrical signals from the user's body. The system can acquire the dynamical eye orientation, which is sent to the micro-controller to control the artificial eye to have the same orientation. The bioelectrical sensors, micro-servomotor and artificial eye model are set up to investigate the eye movement detection and control methods. Different configurations of the electrodes are explored, and the best configuration is used to sense the eye movement and controls the artificial ocular.

EOG is useful in ophthalmology for diagnosis and prognosis of several diseases such as Best's disease (Pinckers et al. 1996) and multiple sclerosis (Prim Espada et al. 1996). It can be used for diagnosis and prognosis of several eye ailments caused by primary depigmentation such as those associated with myopia, siderosis or chloroquine intoxication, and retinopathies due to hypertension or diabetes (Choudhury et al. 1989). Besides the clinical research and laboratory use, EOG is also broadly used in assistive technologies, such as the HMI (human machine interface) (Patmore and Benjamin 1998), which provides a pointing device that could be useful to people with physical disabilities and for the determination of eye position in fast jet flight (Viveash et al. 1994b).

From the above studies, we found out that EOG is simple to use, the electronic parts can be very small and it can be used for long-term monitoring. These characteristics are required for the purpose of our project. Therefore, it was selected as our eye motion sensor.

\section{Using EOG to detect the horizontal eye movement}

Two small (6-8 $\mathrm{mm}$ in diameter) electrodes are used for the recording contact points around the eyes. A disposable electrode with low impedance, functioning as a reference electrode, is located on the midline of the forehead. The configuration of the electrodes is shown in Fig. 4. One pair of horizontally placed electrodes is used to record the horizontal eye movements. To record the one eye horizontal movements, one electrode needs to be moved to the middle point of the two eyes. EOG electrodes can easily be fixed in place without causing any discomfort to the 


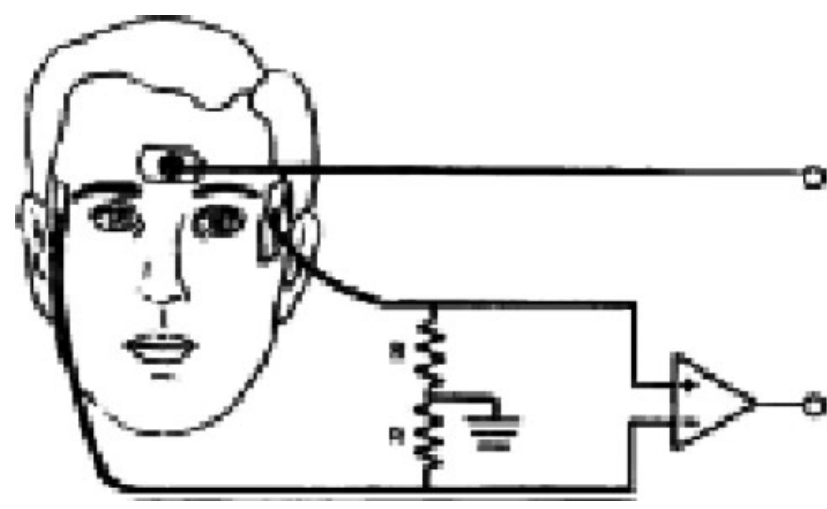

Figure 4 Electrode configuration.

subject. The subject can wear spectacles and the EOG apparatus does not interfere significantly with the observer's visual field.

\section{Using FIR median hybrid filters to remove the eye blink artefact from EOG}

The EOG signal is always corrupted by the eye blink artefact. In this section, we present an improved median filter to remove the eye blink artefact from the EOG signal.

Median filtering, first introduced in 1971, is a rather well-understood, non-linear data smoothing technique useful for noise suppression (Gallagher and Wise 1981; Lee 1996). Median filters are used in many signal-processing applications (Wichman et al. 1990) and in image processing (Zeng et al. 1991) due to their good edge preserving ability combined with moderate noise attenuation on flat regions. A median filter is better than a classical smoothing procedure in certain situations; their advantages are:

(1) median filters preserve sharp edges, whereas linear low-pass filtering blurs such edges;

(2) median filters are very efficient for smoothing of spiky noise.

A numeric example is given in Fig. 5. Input signals are shown in Fig. 5A without noise and in Fig. 5B with white noise. Both signals have the spike, which should be removed. Figures 5AA and 5BB are the corresponding processed results. For the signal without noise, the spike is wiped out, and the signal is totally restored. For the second input with white noise corrupted, the spike is also removed, some very small spike occurred somewhere else, which is related to the window length. In noise-free situations, an ideal edge is preserved completely, the property cannot be achieved with any other linear low-pass filter. However, in noisy conditions the median filters also lose their ability to preserve sharp edges, so an improved FIR median hybrid filter is used instead.

\section{Median filters and improved median filters}

Median filters. The median of $n$ numbers $x_{1}, x_{2}, \ldots, x_{n}$ is, for $n$ odd, the middle number in size. It can be written in the form:
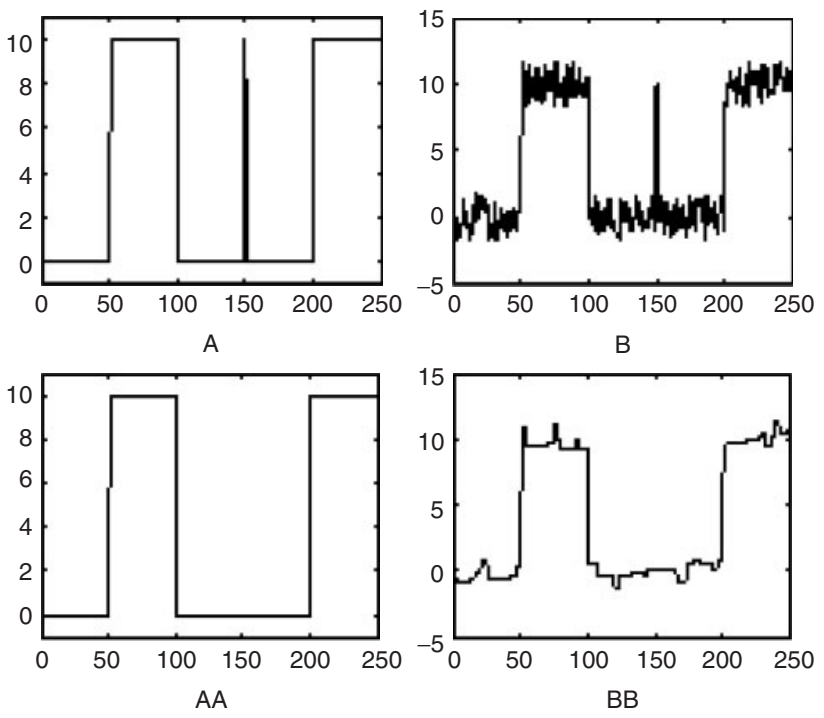

Figure 5 Median filters.

$$
\operatorname{Median}\left(x_{1}, x_{2}, \ldots, x_{n}\right)
$$

A median filter of size $n$ on a sequence is, for $n$ odd, defined through

$$
y_{i}=\operatorname{Median}\left(x_{i}\right)=\operatorname{Median}\left(x_{i-v}, \ldots, x_{i}, \ldots, x_{i+v}\right),
$$

where $v=(n-1) / 2$, median filters are known to preserve sharp changes in signals, which, in many cases, happened in biomedical and communication signals. However, one of the disadvantages of the median filters is the fact that sinusoidal waveforms are distorted in the process, another disadvantage is that the median filters lose their properties in noise conditions. To solve this problem, a hybrid filter is introduced.

FIR median hybrid filter. The FIR median hybrid (FMH) filters consists of a few linear FIR subfilters and the median operation taken over the outputs of the substructures. All kinds of FIR filters can be used as substructures. The general form of the hybrid filter is defined by

$$
y(n)=\operatorname{Median}\left(f_{1}(x(n)), f_{2}(x(n)), \ldots, f_{m}(x(n))\right),
$$

where $f_{1}, f_{2}, \ldots, f_{m}$ are linear FIR filters, which work on the input signals.

The block diagram of the general structure of the FMH filter is shown in Fig. 6.

Least-square optimal approach. When the sensor is used to explore the property of the object, the information obtained must be maximized. The object's property is changing with respect to time. A higher sample rate will be used to detect the object, so that the object property change is insignificant during the time interval. In this way, the data for each sensor are very smooth and can be approximated by a polynomial equation. We are given a collection of data 


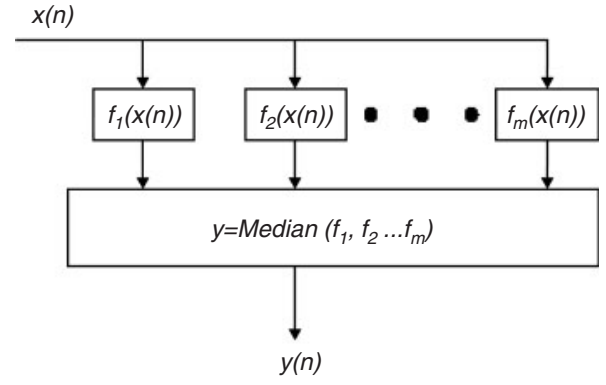

Figure 6 General structure of the FMH filter.

$\left\{z\left(t_{1}\right), z\left(t_{2}\right), \ldots, z\left(t_{n}\right)\right\}$ that are noisy measurements of a process, which are estimated by a polynomial equation and can be expressed as follows:

$$
y=a_{0}+a_{1} t+a_{2} t^{2}+\cdots+a_{n} t^{n},
$$

where $t$ is time, $\left[a_{0}, a_{1}, \ldots, a_{n}\right]$ are unknown coefficients.

Measurements are of

$$
z\left(t_{k}\right)=y_{k}+v_{k},
$$

where $v_{k}$ is an observation error, with variance $\sigma_{k}^{2}$.

We let the estimate be

$$
\widehat{y}_{k}=\widehat{a}_{0}+\widehat{a}_{1} t+\widehat{a}_{2} t^{2}+\cdots+\widehat{a}_{n} t^{n}
$$

Assume that the observation error with zero mean are uncorrelated with each other, and have the variance $\sigma_{k}^{2}$, or the covariance matrix

$$
R=\operatorname{diag}\left\{\sigma_{1}^{2}, \ldots, \sigma_{n+1}^{2}\right\}
$$

The residual is designed as

$$
r_{k}=z_{k}-\widehat{y}_{k}=\left(y_{k}-\widehat{y}_{k}\right)+v_{k}
$$

The mode is shown in Fig. 7.

The least-square estimation problem may be stated: determine $\left\{a_{k}\right\}$ to minimize the sum of the squared weight residuals

$$
\mathcal{F}=\sum_{k=1}^{n}\left(r_{k} / \sigma_{k}\right)^{2}
$$

We can find $\left\{\widehat{a}_{k}\right\}$ by setting

$$
\frac{\partial \mathcal{F}}{\partial\left(\left\{\widehat{a}_{k}\right\}\right)}=0
$$

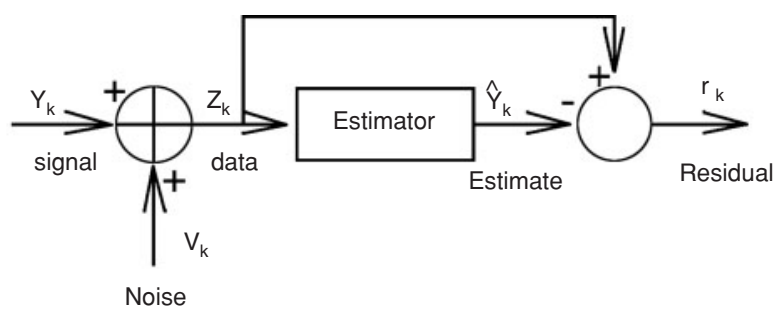

Figure 7 The block diagram of the estimator.

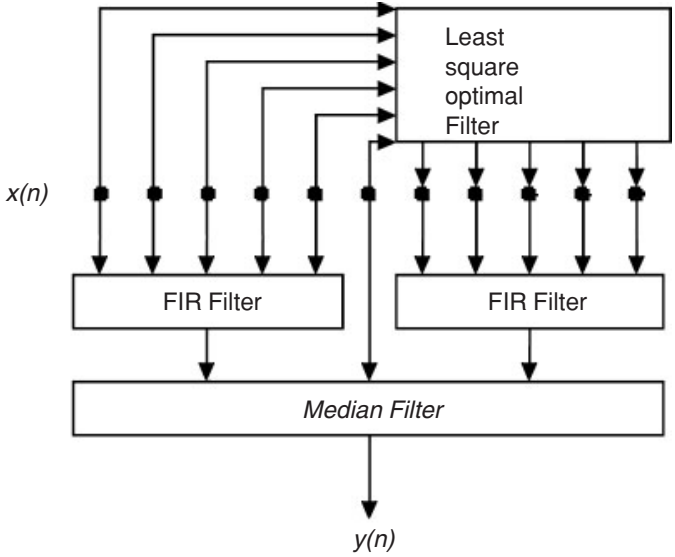

Figure 8 Linear-non-linear combinational filters.

Once we get $\left\{\widehat{a}_{k}\right\}$, we get the model signal, then by plugging the next time interval, we will be able to estimate the next step signal.

Residual smoothing. Small white noise can be filtered out by the residual smoothing method.

Assume that the $x$ variables are generated by a signalplus-noise model

$$
x_{i}=s_{i}+n_{i}
$$

where the signal $s_{i}$ varies slowly compared to the noise $n_{i}$. Median filtering gives an estimate of $s_{i}$

$$
y_{i}=\operatorname{Median}\left(x_{i}\right) \approx s_{i}
$$

Thus the residuals

$$
\widehat{n}_{i}=x_{i}-\operatorname{Median}\left(x_{i}\right) \approx n_{i}
$$

gives the estimates of the noise variables. Further median filtering of the residuals could reduce the noise further

$$
z_{i}=\operatorname{Median}\left(\widehat{n}_{i}\right) \approx 0
$$

Addition of $y_{i}$ and $z_{i}$ now hopefully gives a good estimate of $s_{i}$,

$$
\begin{aligned}
\widehat{s_{i}}=y_{i}+z_{i}= & \operatorname{Median}\left(x_{i}\right) \\
& +\operatorname{Median}\left[x_{i}-\operatorname{Median}\left(x_{i}\right)\right]
\end{aligned}
$$

Simulation results show that using the above equation can achieve better results than using equation (13).

Linear-non-linear combinational filters. A new class of linear-non-linear combinational filter is developed to restore the biomedical signal not only from the impulsive noise, but also from the Gaussian noise. Figure 8 shows the structure of the linear-non-linear combinational filter. It consists of two parts. The least-square optimal filters are used in the first layer to remove the noise. The median filter is used in the second layer to preserve the edge and remove the impulses. Better performance can be achieved by using the following structured filter. It can remove the impulse noise, preserve the edge and suppress the white noise. 

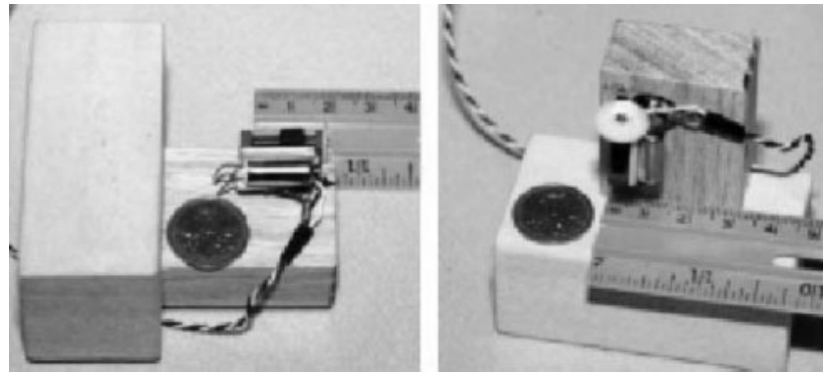

Figure 9 Light servomotor is compared with a penny.

\section{TECHNICAL ASPECTS OF EXPERIMENTAL SYSTEM}

\section{Motor}

A small servomotor has been used to drive the artificial eye. The motor shown in Fig. 9 is one of the lightest normal servos, weighing only $3.5 \mathrm{~g}$. With a $5-\mathrm{V}$ power supply, the motor has sufficient torque to drive the artificial eyeball mounted onto the motor shaft. A servomotor is controlled by a pulse-modulated signal. The width of the pulse is the code that signifies to what position the shaft should turn.

\section{ECG (Electrocardiography) electrode}

The electrode is an ECG silver/silver chloride electrode, which is used to detect the ECG. The disposable electrode is about $1 \mathrm{~cm}$ in diameter and attached to the rectangular disposable sponge pads. The tip of a 30-in. lead wire is attached to the electrode. It can also be used to detect EOG, which measures the electrical potential of the eyes. The human eye is a dipole. The EOG signal can be used to measure the positions of the eyes with respect to the head. The electrical axis of the eye corresponds to its visual axis. Determining orientation of the dipole will determine the orientation of the eye. The EOG can record the eye movements over $70^{\circ}$, with a typical accuracy of approximately $\pm 1.5^{\circ}$ to $\pm 2^{\circ}$. However, greater resolution is possible by averaging equivalent responses.

\section{Artificial eye model}

An eye pit model is used in the experiment as shown in Fig. 10. The pit size of the model is the same size as the natural eye pit. Eyeballs are mounted on to the servomotors.

\section{Infrared reflection based eye movement detection system set-up}

Multiple sensors are used to detect the eye movement $(\mathrm{Gu}$ et al. 1999). A nine-cell pair infrared sensor array is used to detect the eye movement. Herein only a three-cell sensor array is used for the detection. Experimental study has been carried out using the artificial eye model, as shown in Fig. 11. Two artificial eyeballs are mounted inside the eye socket model, which have the same approximate volume

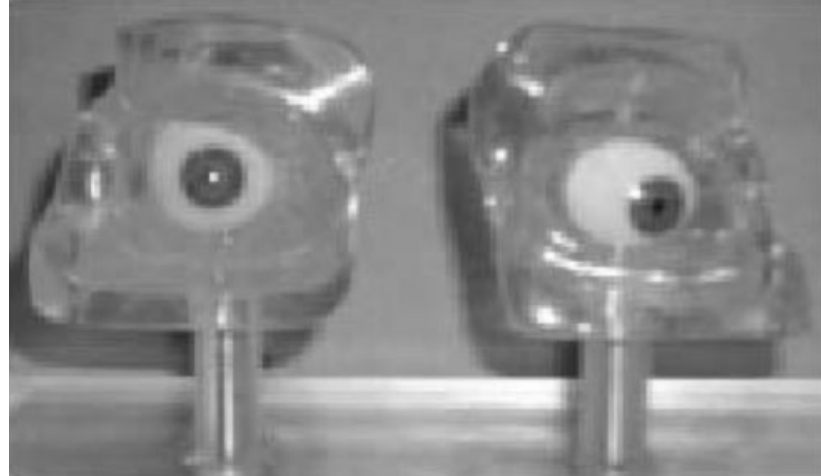

Figure 10 Artificial eye model.

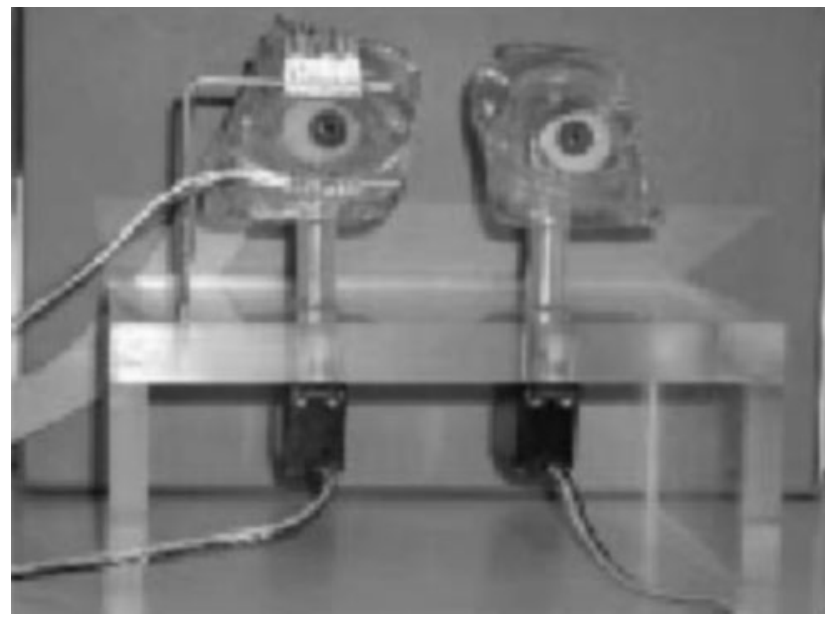

Figure 11 Experimental set-up.

as the real eye pit. The two eyeballs are linked to the servomotors, which are controlled by a micro-controller.

The micro-controller drives the servomotor to move the eyeball using a predefined eye movement signal. The infrared emitter will send out the infrared light to illuminate the artificial eye, and the infrared detector will receive the reflected infrared light.

\section{EOG-based eye movement detection system set-up}

The EOG is usually recorded as a direct current (DC) signal because the movements of the eyes are relatively slow in both electrical and electrophysiological terms. The potential differences between the two electrodes are amplified and sent to the computer through the AD card with a 30-Hz sample frequency. At the same time, the amplified signal is shown on the screen of an oscilloscope, as shown in Fig. 12.

\section{EXPERIMENTAL RESULTS}

\section{Experimental results for neural network based sensor fusion}

\section{Sensor data space creation}

The relationship between the infrared array output and the eye position is non-linear. Training the neural network 


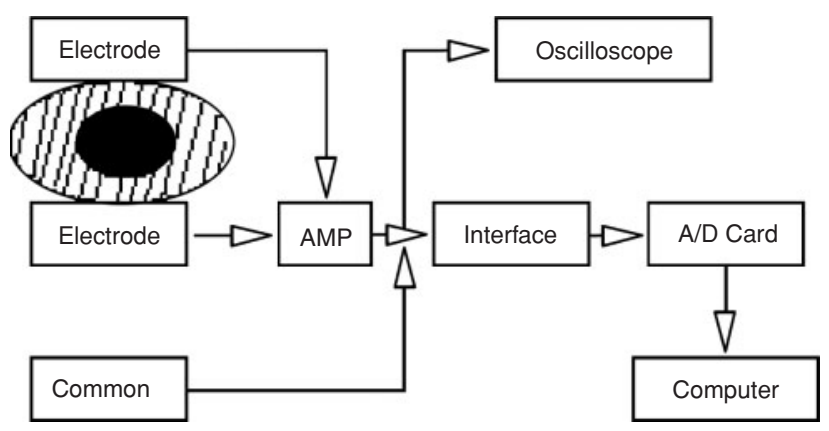

Figure 12 The block diagram of EOG-based eye movement detection system.

could complete the non-linear mapping between the input and the output; this is divided into the following three steps:

1. Calibration: The first step is to let the eye move following the predefined eye movement signal and record the infrared sensor output simultaneously. This calibration procedure is repeated until the number of times is large enough to minimize the expected square error.

2. Training: The recorded sensor output is used as the input for the multi-layer neural network and the predefined eye movement signal as the output to train the supervised network. The weight and the bias of the network are obtained as the information for the mapping between the sensors and the eye position.

3. Experiment: During the experiment, feed the recorded sensor output to the neural network, the output of the network is the eye movement signal.

As shown in Fig. 13, the artificial eyeball movement range is $40^{\circ}$, from $-20^{\circ}$ to $+20^{\circ}$. The servomotor drives the artificial eyeball from the left end to the right end at slow speed. The resolution is $1^{\circ}$ for the time being. The infrared sensor then recorded the data and sent it to the computer

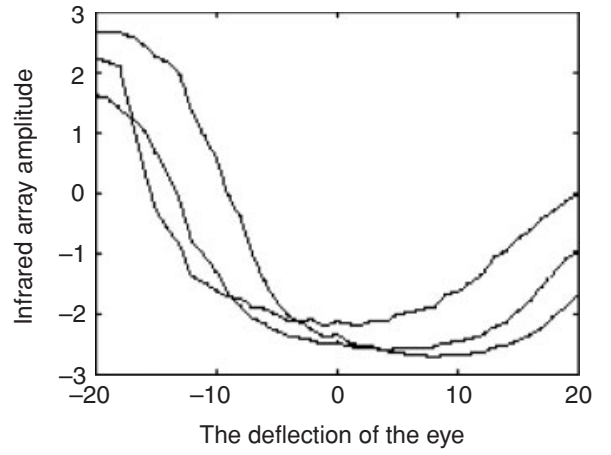

Figure 13 The eye movement record in tracking target.

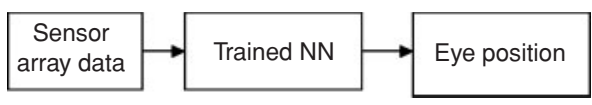

Figure 14 The fusion block diagram.

for analysis. Using the three steps described above, the trained neural network is obtained.

\section{Experimental results for fusion}

For the sensor fusion experiment, the periodical eye movement signal is sent to the controller to drive the artificial eyeball. The recorded sensor array data with noise are fed into the trained neural network to get the fusion output. Figure 14 shows the process.

\section{Experimental results for fault-free sensor data}

MATLAB is used for neural network training and simulation. Figure 15 shows some experimental results for sensor fusion.

The left panel of Fig. 15 shows the infrared array data. From the figure, we can see that the artificial eye moves back and forth three times during the experimental study. The right panel of Fig. 15 shows the eye position output. The result verifies the fusion algorithm.
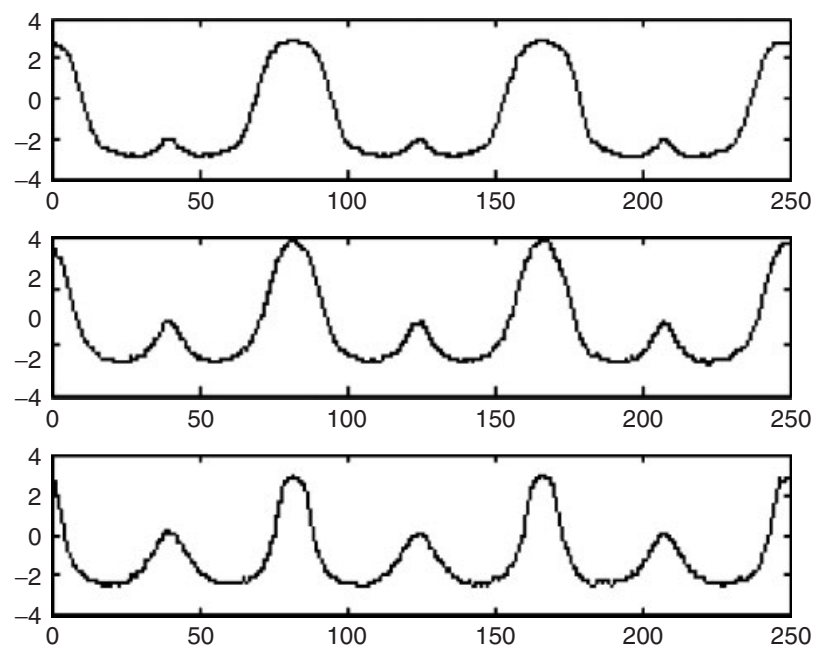

Figure 15 Fusion with fault-free data.

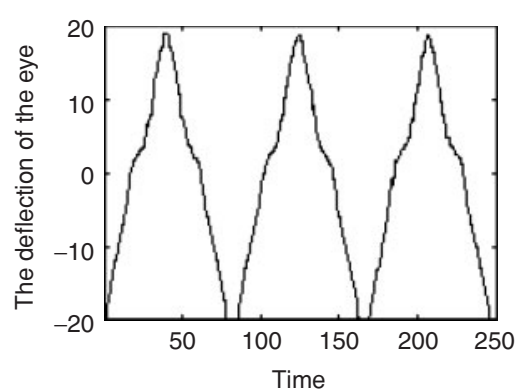




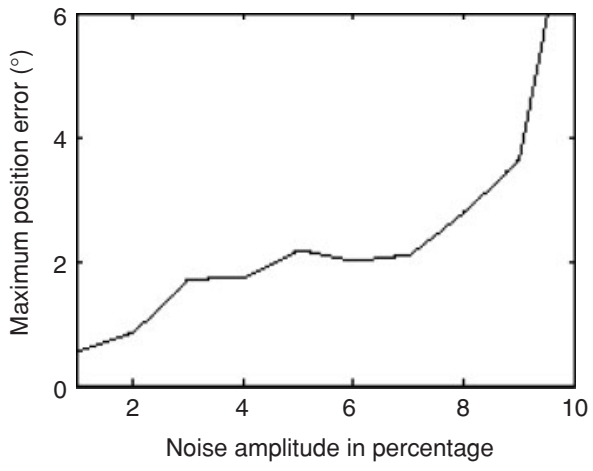

Figure 16 Fusion with soft sensor failure.

\section{Experimental results for soft sensor failure}

Soft sensor failure means that the sensors are still working; however, there will be some noise in the sensor data, such as the bias, drifting and precision degradation. The amplitude of the sensor noise is very low. Figure 16 shows the experimental results with soft sensor failure. In the experiment, the random noise is added to the sensor output. The noise amplitude is from $1 \%$ of the maximum sensor output amplitude to $10 \%$ of the maximum amplitude. The relationship between the noise amplitude (as a percentage) and the position error (in degrees) is shown in the figure.

\section{Experimental results for hard sensor failure}

Hard sensor failure means that the sensor does not work at all. Usually in electronics it can be defined as the "stuckat" sensor failure, where the sensor is stuck at one extreme of its signal range. In practice, this is likely to be an open (stuck-at 0) sensor or a short circuit (stuck-at 1) sensor. Figure 17 shows results when sensor cell one, cell two and cell three stuck at 0 , respectively. It is clear that using the trained network without failure detection and recovery, the result is totally meaningless.

\section{Experimental results for failure detection and recovery}

To deal with the sensor failure, two types of neural networks have been generated. The training steps of the neural networks is described as follows:

Sample selection: Select a set of data samples for normally working sensors and a failed sensor, whose readings are to be recovered.

Training for failure detection network: Using the data from the normally working sensor and the faulty sensor data as input for the multi-layer neural network, the maximum and minimum values are assigned as output for those two types of data, respectively, to train the supervised network. Training for failure recovery network: Use the output from the normally working sensor as input for the multi-layer neural network and the failed sensors' data as output to train the supervised network. The weight and the bias of the network are obtained as the information for the mapping between the normal sensors and the abnormal sensors.
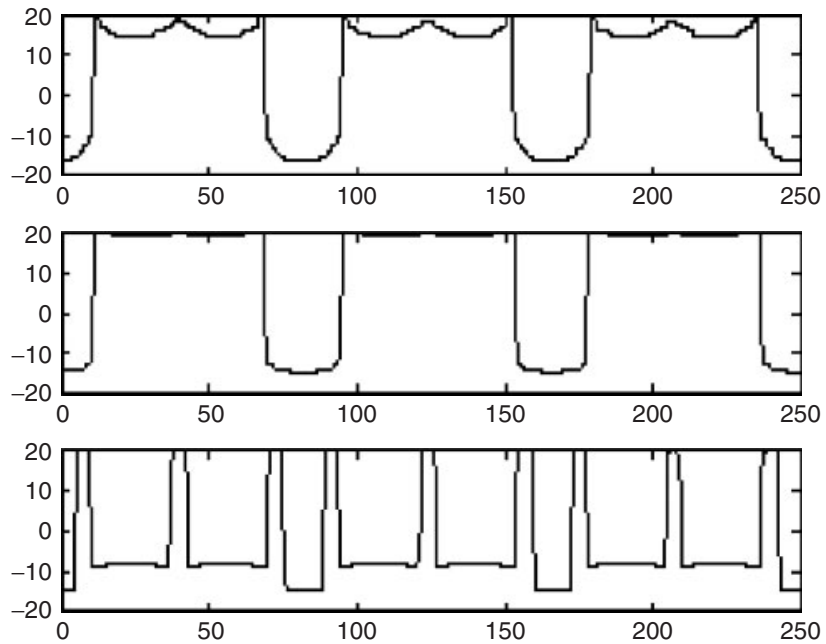

Figure 17 Hard sensor failure without failure detection and recovery.

Completion: Try to add the sets of normal sensor versus abnormal sensor data sample. Thorough exploration of the data will enable the network to deal with different sensor failure configurations.

Using the trained neural network, the previous hard failure problem can be solved easily. As shown in Fig. 18, the left side is the detection result. The threshold distinguishes the normal sensor data and the abnormal sensor data. The right panel shows the recovered eye position signal.

\section{Experimental results for EOG eye movement detection}

\section{Sensor placement}

When using EOG to detect eye movement, it is important to know where to apply the electrodes. To detect the horizontal eye movement, one pair of electrodes is placed on the side of the eye, and the reference electrode is put on the forehead. The question is if the two electrodes placed on the side of the eye have vertical offset, will it affect the result of the EOG. Experimental study has been carried out by trying different configurations of the electrodes. Three pairs of electrodes are placed on the side of the eye, as shown in the Fig. 19. The pair with no vertical offset with respect to the eye is called the middle pair. The upper one is called the up pair and the lower pair is called the down pair.

For three pairs of electrodes, there are a total of nine configuration sets, i.e. up-up, up-middle, up-down, middleup, middle-middle, middle-down, down-up, down-middle and down-down. For each configuration, the subject is asked to fixate at a centre point throughout the test. The recorded data for each set are plotted in Fig. 20. It is clear from Fig. 20 that the middle-middle pair of electrodes has the lowest drift. This is verified by comparing the variance in Table 1 , which shows that the middle-middle pair has the lowest variance. From the experiment, it is also found that the eye blink artefact shows up in EOG 

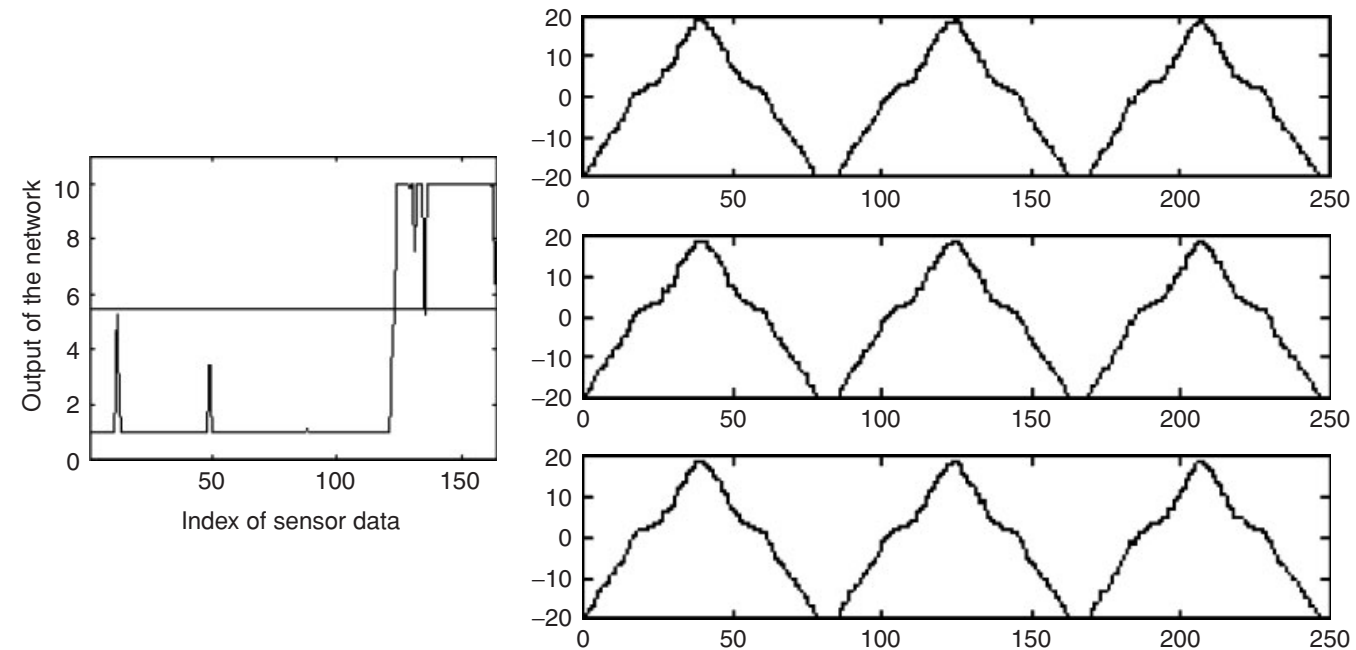

Figure 18 Failure detection and recovery.

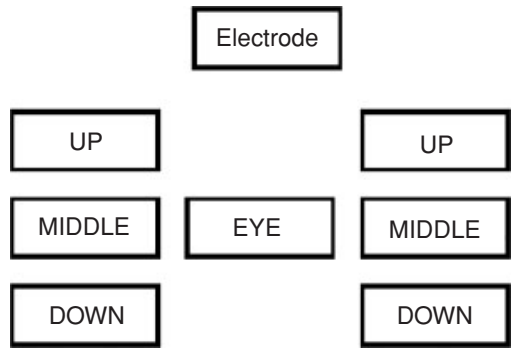

Table 1 The variance of the data from each set of configuration

\begin{tabular}{llll}
\hline & $\mathrm{Up}$ & Middle & Down \\
\hline $\mathrm{Up}$ & 0.2581 & 0.0810 & 0.0544 \\
Middle & 0.5624 & 0.0038 & 0.0270 \\
Down & 0.1879 & 0.0470 & 0.0437 \\
\hline
\end{tabular}

Figure 19 The configurations of the electrodes.

signals. The eye blink signal has less effect on the EOG signal that uses the middle-middle pair, but corrupts all the rest of the sets. The eye blink signals are the small spikes in the wave of the signal, and is easy to see in each set of data.
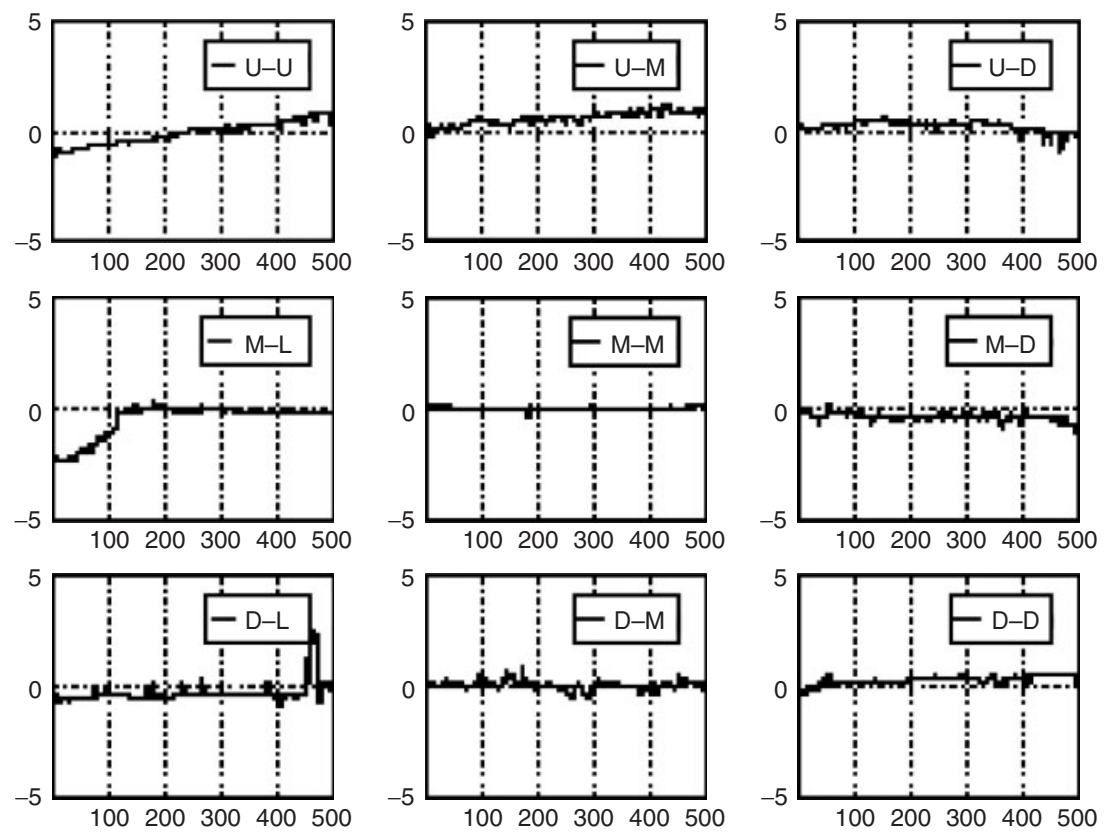

Figure 20 The baseline EOG to detect eye movement with different electrode configurations. U: up, D: down, M: middle. The subject is asked to focus on a point at the centre of the target. 

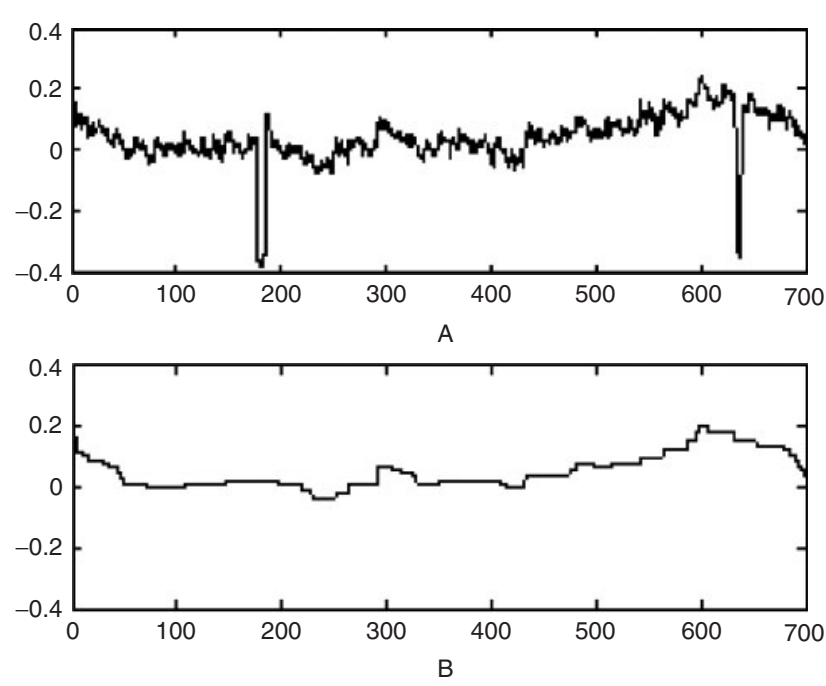

Figure 21 Using the median filter to remove the eye blink.

\section{Using the median filter to remove the eye blink from the corrupted signal}

The overall performance of the EOG detection system is good. The subject is asked to sit still and quiet to prevent the EMG and other artefacts from entering the EOG channel. During the experiment, the subject is asked to look at the centre first and intentionally blinked his/her eye. Then the subject randomly moved his/her eye. The EOG signal will be recorded. In Fig. 21, the upper panel is the eye blink-corrupted EOG signal. It is apparent that two eye blinks occurred during the recording period. The filter can remove the eye blink and recover the eye movement signal, as shown in the lower panel.

\section{Two-eye and one-eye EOG signal}

Using the best configuration from the previous section, the two-eye movement and one-eye movement data are recorded. The eye blink artefact is removed by the filter, and the results plotted in Fig. 22. The left panel depicts the two-eye horizontal movement signals. The electrodes are placed close to the temple of each side of the head. The right panel shows the left eye horizontal movement signals. The reason for recording the one-eye movement signal is to find the difference between the one-eye
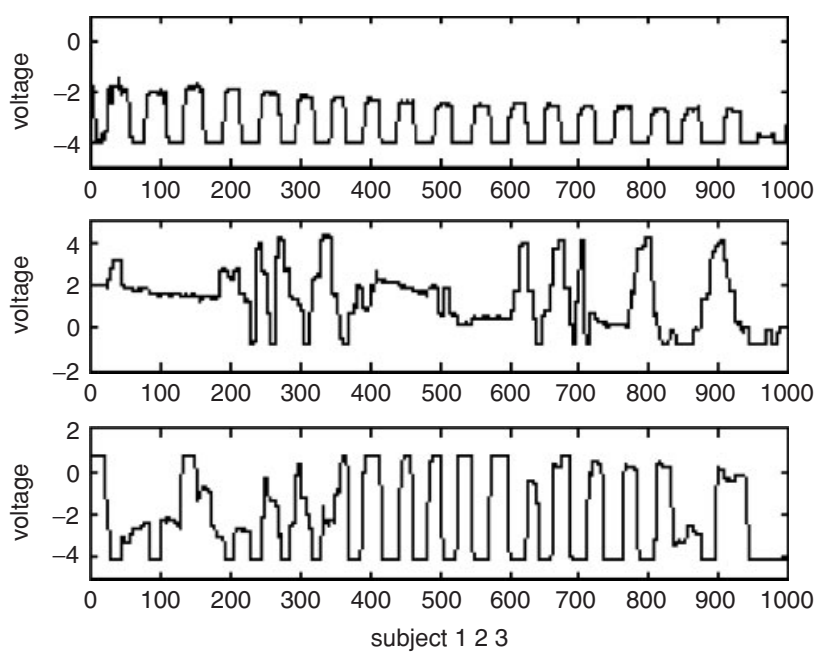

Figure 23 Experimental study with subjects 1, 2 and 3 (from top to bottom).

horizontal movement signal and the two-eye horizontal movement signal. It is clear that the amplitude of the twoeye signal is almost twice that of the one-eye signal. These two figures show the smooth pursuit movement of the eye from the left side to the right side, and back to the left side.

The signal is close to linear, but DC drift is a problem. This is the drawback associated with the EOG, since it is subject to a slow shift in baseline electrical potentials. The drift is primarily caused by the polarization of electrodes.

The following steps are used to improve the signal quality by suppressing the drift:

Preparation. The drift is usually caused by an accumulation of electrical charge in the recording electrodes, but it can be minimized, or even eliminated by several methods through recording contact area site preparation (i.e. mild skin abrasion, conductive paste).

Polarization of the electrodes. Let the subject settle for at least $1 \mathrm{~h}$ or so to ensure the completion of the polarization. Filtering. Using a low-pass filter with the corner frequency set to a very low frequency around $10 \mathrm{~Hz}$.

Using the methods above, low drift EOG signals can be obtained as shown in Figs. 23-25. The subject is required to direct his gaze from left to right and right to left
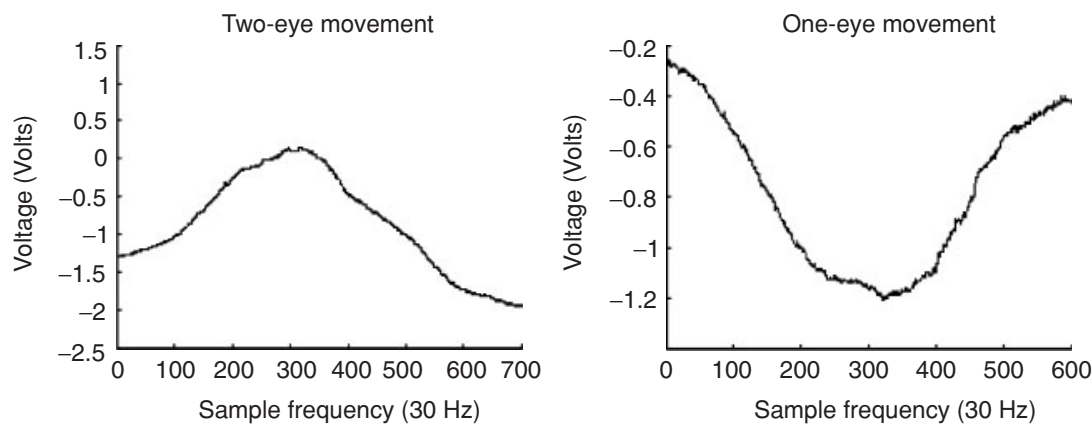

Figure 22 Using the EOG to detect one-eye (one electrode at nose and one at temple) and two-eye movement (electrodes at each temple). (The subject moved his/her eye from one extreme to another and moved back.) 

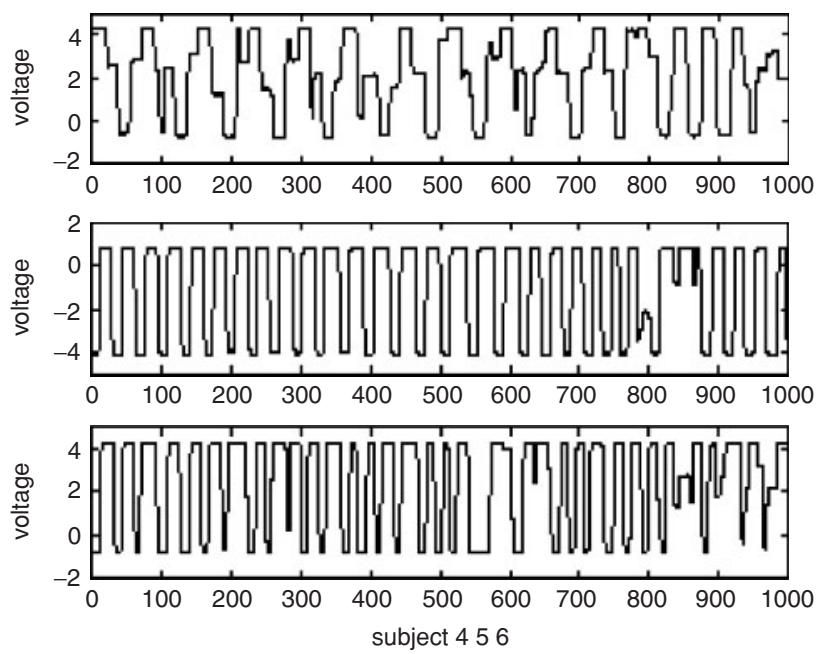

Figure 24 Experimental study with subjects 4, 5 and 6 (from top to bottom).

very quickly. The experimental data of the first subject are shown at the top of Fig. 23. Drift can be observed from the EOG signal, possibly because the long leads are used in the experiment. When the long leads are replaced by the short leads, there is minor drift in the second subject's EOG signal (middle plot, Fig. 23). From the EOG signal of the third subject (bottom plot, Fig. 23), we found that the signal is bounded at the bottom and top. The reason for this is that the amplifier gain is tuned a little bit high during the experiment; this also happened in Figs. 24 and 25.

Experimental study with the eye movement and motor control The EOG is used to determine the natural eye position signal, which is used to control the artificial eye movement. To find out the relationship between the output of the sensor and the eye deflection angle, a calibration set-up is necessary, as shown in Fig. 26.
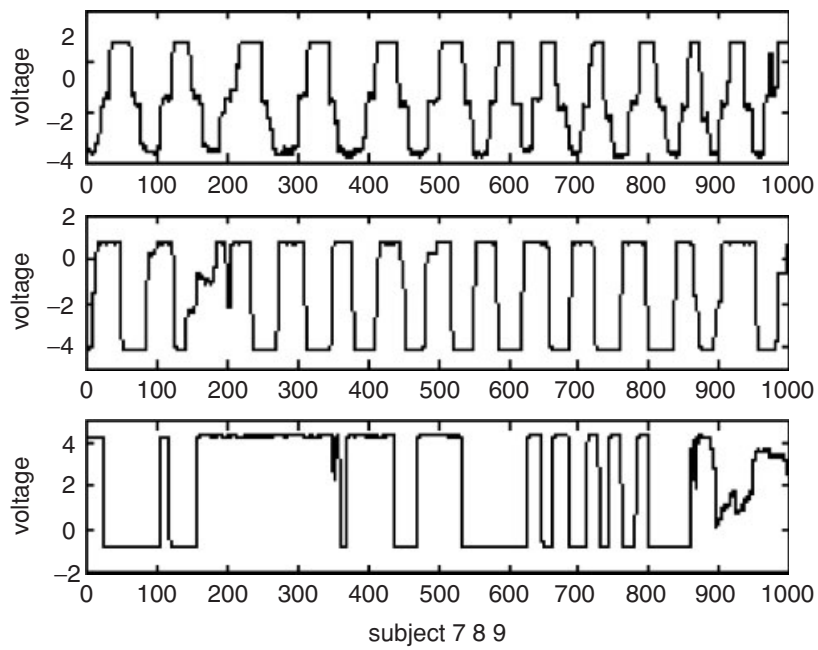

Figure 25 Experimental study with subjects 7, 8 and 9 (from top to bottom).

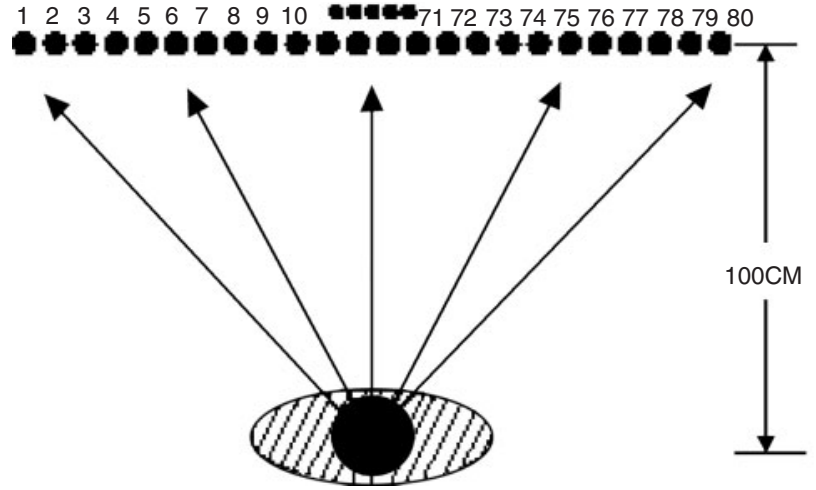

Figure 26 The natural eye calibration set-up.

The subject is asked to stabilize his/her head in front of a board. The distance between the neighbour targets is constant. The distance between the subject and the board is set to $1 \mathrm{~m}$. The deflection angle of the subject's eye is determined from the distance between the start target dot and the end target dot. During the experiment, the subject is asked to look to the left, right and to the centre of the target board. Then the subject is asked to move his/her eye horizontally by looking at specific targets. Assume that the subject's left end vision is $T_{\mathrm{L}}$, right end is $T_{\mathrm{R}}$, and centre $T_{\mathrm{C}}$. The distance between the subject and the target board is $L$. Then the subject's eye deflection range is

$$
\text { Range }=\arctan \left(T_{\mathrm{C}}-T_{\mathrm{L}}\right) / L+\arctan \left(T_{\mathrm{R}}-T_{\mathrm{C}}\right) / L
$$

The subject is asked to fixate at the left-end target, and move the eye to the next target until he/she reaches the right-end target. The corresponding voltage is recorded and a calibration curve generated, as shown in Fig. 27. The deflection range of the subject here is $102^{\circ}$. The calibration curve is quite linear from $-30^{\circ}$ to $+40^{\circ}$.

After the calibration curve is obtained, the eye movement signal is fed to the micro-controller to control the artificial eye model, as shown in Fig. 28. The subject is asked to track the moving target with his/her eye. The eye position signal is fed to the controller to move the artificial eyeball so that it will have the same line of gaze as the natural eye. A pilot study has been carried out. The whole

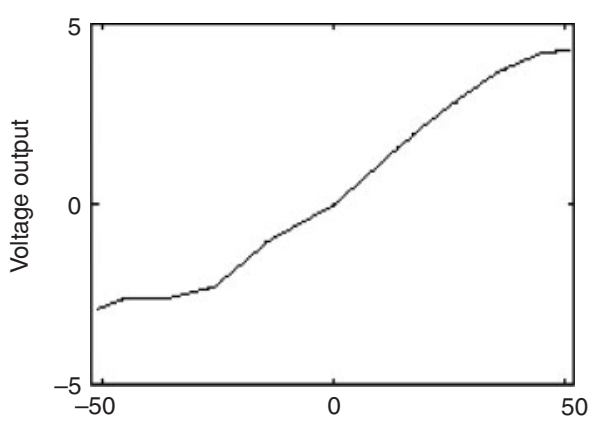

Figure 27 The calibration curve of the EOG to detect eye movement. 


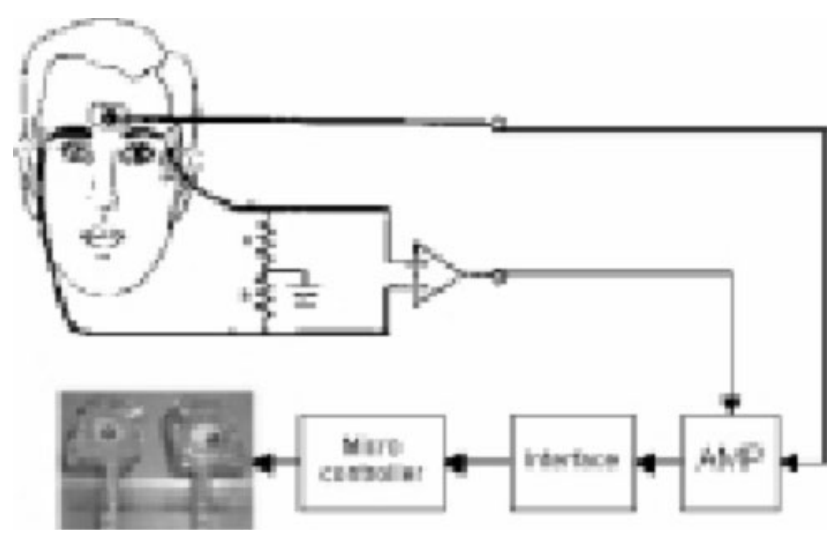

Figure 28 The eye movement detection and ocular system set-up.

procedure has been videotaped and promising results obtained.

\section{CONCLUSION AND FUTURE DIRECTIONS}

In this paper, several aspects of eye movement detection and motor control are investigated. The results can be summarized as follows.

A neural network based approach for sensor fusion is developed for the multiple sensor fusion. An artificial neural network can learn the characteristics of a non-linear, nonmodelled system through training samples. Then during the real application, the sensor signal can be used to feed the network and obtain the desired output.

A newly developed intelligent sensor and control system is described. This is the second-generation model of the robotic eye project, which comprises biomedical electrodes and a micro-controller. The system is intended to provide a rehabilitation ocular implant device that could be useful to some people with ocular implants. With the proposed device, the ocular implant can have the same natural movement as the normal eye. The basis for this system is the use of bioelectrical signals from the user's body. The system can acquire the dynamical eye orientation, which is sent to the micro-controller to control the artificial eye to have the same orientation. The bioelectrical sensor, micro-servomotor and artificial eye model are set up to investigate the eye movement detection and control methods. The pilot study has demonstrated its potential for clinical applications.

A new approach of using FIR median hybrid filters for analysis of eye tracking movements is described. The program can filter out the eye blink artefact from the eye movement signal. Because the eye blink artefact is always corrupted in a EOG signal, it must be filtered out for the purpose of our project. The FIR median hybrid filter is studied, and its properties are explored with examples. Finally the filter is used to deal with a real eye blinkcorrupted EOG signal. Examples of the analysis procedure for eye tracking or a random moving target are given. The method has proved to be highly reliable.

\section{Future works}

The work presented in this paper can only be considered preliminary, since many challenging and possibly more important problems have not been covered in this paper. In this section, a number of problems will be proposed as future directions for research.

Circuit minimization is very important in the design of this robotic eye system. Because the whole system, the motor, the artificial eyeball and the micro-controller will be integrated into the size of the eye pit volume, it is critical to find a tiny, powerful and energy efficient motor for the system. The controller not only should be small, but also should be able to process the sensor signal and control the motor to drive the eyeball to move simultaneously with the real eye. Future directions for research in this project include searching for a smaller motor, trying out a smaller sized controller and necessary components, and integrating them into a small size system.

Energy efficiency is a very important issue in the further design of the robotic eye system. The motor used to drive the eyeball will consume lots of power, so a low power design technique (Liqiong et al. 2000; Mahapatra et al. 2000 ) has to be incorporated into the system. One approach is to search for a high power, rechargeable and smaller size battery. In this case, the whole system should be very easily removable. There are many high technology rechargeable solutions (Gyu and Cho 1998; Foo et al. 1999), such as infrared charge and microwave charge, which may be taken into consideration.

The sensor is always the key to the success. Although the second generation of the system can detect the eye movement and control the motor to drive the artificial eye to move accordingly, the robustness and the stability of the system need to be further tested and improved. A further research area for eye movement detection may be the residual signal of the injured eye socket, and other multisensor fusion techniques.

\section{ACKNOWLEDGMENTS}

This work was supported by the Natural Science and Engineering Research Council of Canada (NSERC). The authors would like to thank the reviewers for their comments and editorial suggestions.

\section{REFERENCES}

Barschdorff D, Gerhardt D. 1996. Rapid eye movement detection in infants using a neural network. In 18th Annual International Conference of the IEEE Engineering in Medicine and Biology Society, Vol. 1, p. 935-36.

Choudhury JK, Bhattacharya R, Basak TK. 1989. Design and development of electro-oculograph. 7 Instrum Eng India EL, 70(5):166-8.

Chung D, Merat FL. 1996. Neural network based sensor array signal processing. In Proceedings of the 1996 IEEE/SICE/RSJ International Conference on Multisensor Fusion and Integration for Intelligent Systems, p. 757-64. 
Foo CF, Tseng KJ, Zhao L. 1999. New structure transcutaneous transformer for totally implantable artificial heart system. Electron Lett, 35(2):107-8.

Gallagher NC, Wise GL. 1981. A theatrical analysis of the properties of median filters. IEEE Trans Acoust Speech Signal Process, 29(6):1134-40.

Garvey TD, Lowrance GD, Fischler MA. 1981. An inference technique for integrating knowledge from disparate sources. In Proceedings of the 7th International Joint Conference on Artificial Intelligence, p. 319-25.

Gu J, Meng M, Cook A, Faulkner MG. 1999. Sensing and control system for ocular implant. In Proceedings of the 1999 IEEE Canadian Conference on Electrical and Computer Engineering, p. 1408-12.

Gyu BJ, Cho BH. 1998. An energy transmission system for an artificial heart using leakage inductance compensation of transcutaneous transformer. IEEE Trans Power Electron, 13(6):1013-22.

Hackett JK, Shah M. 1990. Multi-sensor fusion: A perspective. In Proceedings of the 1990 International Conference on Robotics \& Automation, p. 1324-30.

Hatzilabrou GM, Greenberg N, Sclabassi RJ, Carroll T, Guthrie RD, Scher MS. 1994. A comparison of conventional and matched filtering techniques for rapid eye movement detection of the newborn. IEEE Trans Biomed Eng, 41(10): 990-5.

Heinonen P, Neuvo Y. 1988. FIR-median hybrid filters with predictive FIR substructures. IEEE Trans Acoust Speech Signal Process, 36(6):892-9.

Jung KC, Chao J, Jang LW. 1996. Uncertain information fusion using belief measure and its application to signal classification. In Proceedings of the 1996 IEEE/SICE/RSJ International Conference on Multisensor Fusion and Integration for Intelligent Systems, p. 151-7.

Kabre H. 1996. On the active perception of speech by robots. In Proceedings of the 1996 IEEE/SICE/RSJ International Conference on Multisensor Fusion and Integration for Intelligent Systems, p. 765-74.

Kandel ER, Schwartz JH, Jessell TM. 1995. Essentials of Neural Science and Behaviour. Norwalk: Appleton \& Lange, p. 571-83.

Lee S. 1996. Sensor fusion and planning with perception-action network. In Proceedings of the 1996 IEEE/SICE/RSJ International Conference on Multisensor Fusion and Integration for Intelligent Systems, p. 687-96.

Liqiong W, Roy K, De VK. 2000. Low voltage low power CMOS design techniques for deep sub-micron ICs. In 13th International Conference on VLSI Design, Vol. 28, p. 24-9.

Mahapatra NR, Garimella SV, Takeen A. 2000. Efficient techniques based on gate triggering for designing static CMOS ICS with very low glitch power dissipation. In 2000 IEEE International Symposium on Circuits and Systems, Vol. 28, p. 537-40.

Martin O, Clodius L. 1979. The history of the artificial eye. Ann Plastic Surg, 3(2):168-71.
Patmore DW, Benjamin KR. 1998. Toward an EOG-based eye tracker for computer control. In Annual ACM Conference on Assistive Technologies, p. 197-203.

Pinckers A, Cuypers MH, Aandekerk AL. 1996. The EOG in Best's disease and dominant cystoids macular dystrophy. Ophthalmic Genet, 17(3):103-8.

Prim Espada MP, De Diego Sastre JI, de Sarria Lucas MJ. 1996. EOG findings in patients with multiple sclerosis. Acta Otorrinolaringol Esp, 47(1):29-31.

Sauter D, Martin BJ, Di Renzo N, Vomscheid C. 1991. Analysis of eye tracking movements using innovations generated by a Kalman filter. Med Biol Eng Comput, 29(1):63-9.

Varri A, Hirvonen K, Hahhinen V, Hasan J, Loula P. 1996. Nonlinear eye movement detection method for drowsiness studies. Int $\mathcal{7}$ Biomed Comput, 43:227-42.

van Dam JWM, Krose BJA, Groen FCA. 1996. Adaptive sensor models. In Proceedings of the 1996 IEEE/SICE/RSJ International Conference on Multisensor Fusion and Integration for Intelligent Systems, Vol. 1, p. 705-12.

Viveash JP, Belyavin AJ, Bigmore DJ, Clarkson GJ, Mccarthy GW, Rumbold DA, Stott JRR. 1994a. Determination of eye position in fast jet flight. Proc SPIE, 2218:78-81.

Viveash JP, Belyavin AJ, Bigmore D, Clarkson GJ, Mccarthy GW, Rumbold DA, Stott JR. 1994b. Determination of eye position in fast jet flight. Proc SPIE, Int Soc Opt Eng, 2218:120-5.

Wang EPX-G, Qian WH, Pei RQ. 1996. On the uncertainty and ignorance of statistical decision and evidence combination. In Proceedings of the 1996 IEEE/SICE/RSJ International Conference on Multisensor Fusion and Integration for Intelligent Systems, p. 166-73.

Wichman R, Astola JT, Heinonen PJ, Neuro YA. 1990. FIR median hybrid filters with excellent transient response in noisy conditions. IEEE Trans Acoust Speech Signal Process, 38(12):892-9.

Wide P, Driankov D. 1996. A fuzzy approach to multi-sensor data fusion for quality profile. In Proceedings of the 1996 IEEE/SICE/RSJ International Conference on Multisensor Fusion and Integration for Intelligent Systems, pp. 215-21.

Yagi T, Uchikawa Y, Kuno Y. 1996. Development of a fish-eye VR system with human visual functioning and biological signals. In Proceedings of the 1996 IEEE/SICE/RSJ International Conference on Multisensor Fusion and Integration for Intelligent Systems, Vol. 1, p. 389-94.

Yuan LX, Wang A, Zheng N, Fu X. 1996. Multiplicative inhibitory velocity detector (MIVD) and multivelocity motion detection neural network model. In Proceedings of the 1996 IEEE/SICE/RSJ International Conference on Multisensor Fusion and Integration for Intelligent Systems, p. 476-83.

Zeng B, Zhou H, Neuro Y. 1991. Weight FIR median hybrid filters for image processing. In 1991 International Conference on Circuits and Systems, Vol. 1, p. 793-6.

Zheng YJ, Bhanu B. 1996. Adaptive object detection from multi sensor data. In Proceedings of the 1996 IEEE/SICE/RSJ International Conference on Multi sensor Fusion and Integration for Intelligent Systems, p. 633-40. 

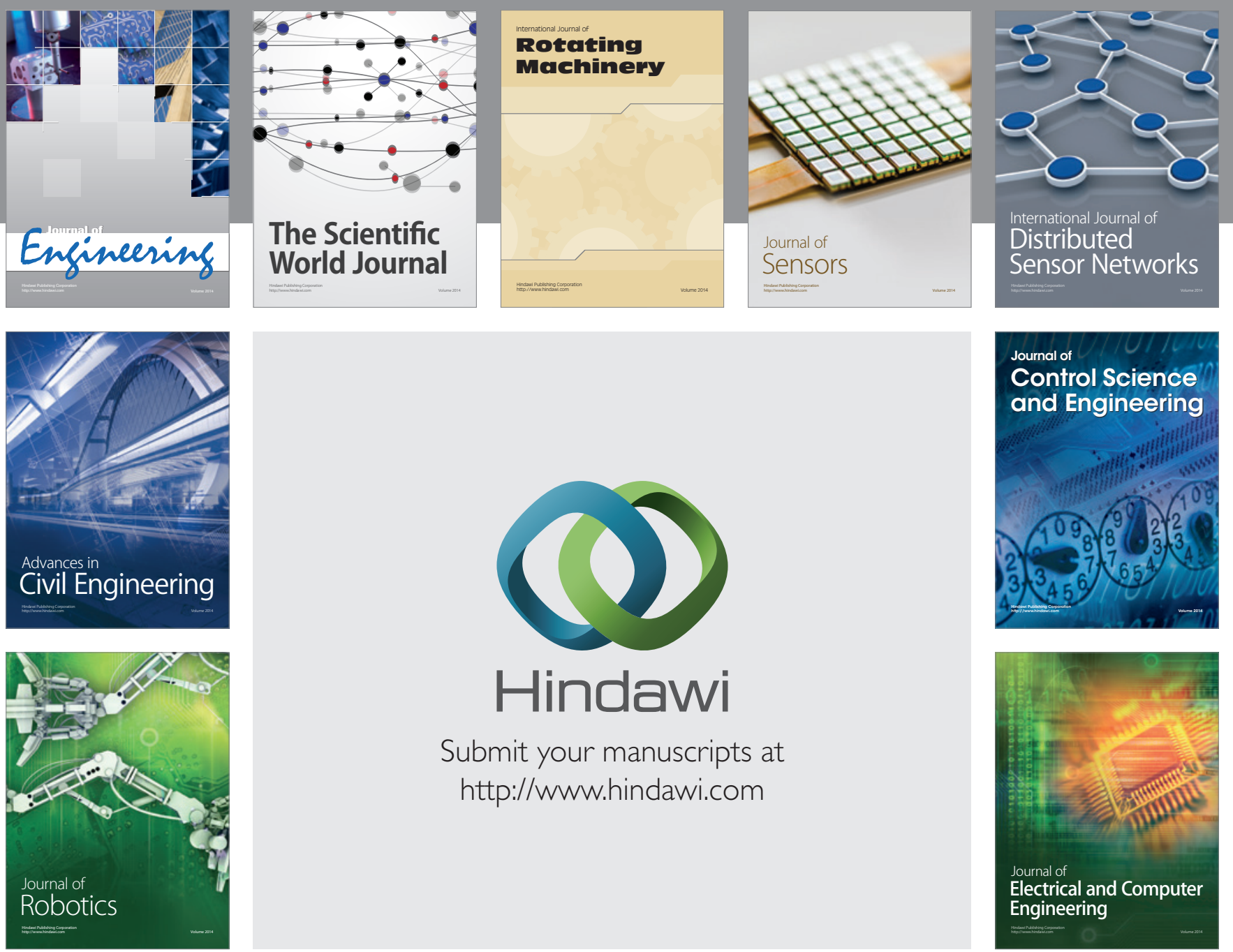

Submit your manuscripts at

http://www.hindawi.com
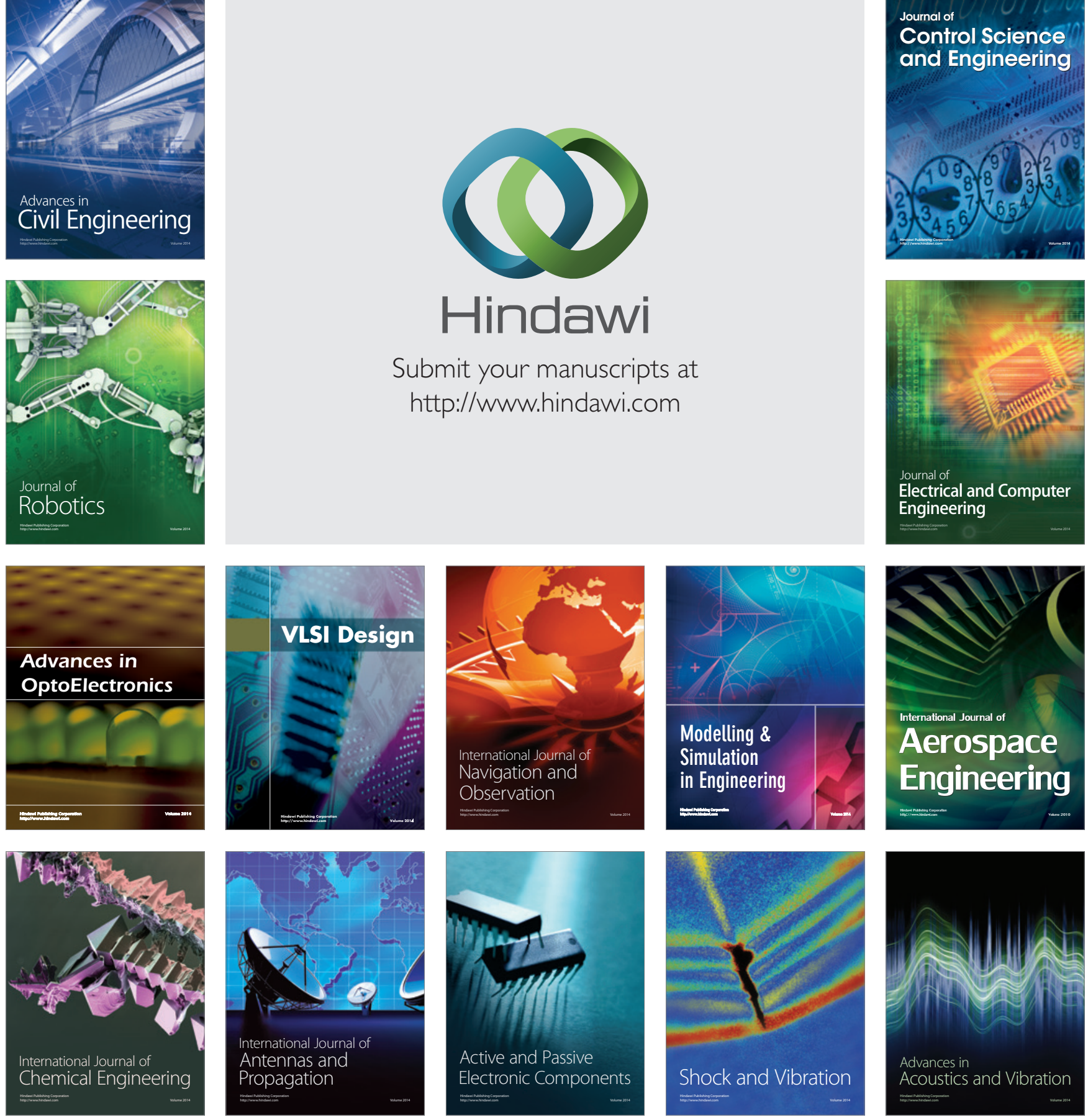